\title{
Ortho-para conversion in the solid hydrogens at high pressures
}

\author{
M.A. Strzhemechny ${ }^{1,2}$ and R.J. Hemley ${ }^{2}$ \\ ${ }^{1}$ B. Verkin Institute for Low Temperature Physics and Engineering \\ of the National Academy of Sciences of Ukraine, 47 Lenin Ave., Kharkov 61103, Ukraine \\ ${ }^{2}$ Geophysical Laboratory and Center for High-Pressure Research, Carnegie Institution of Washington \\ 5251 Broad Branch Road NW, Washington DC 20015, USA \\ E-mail: strzhemechny@ilt.kharkov.ua
}

\begin{abstract}
At low pressures the ortho-para conversion in $\mathrm{H}_{2}$ and $\mathrm{D}_{2}$ is a slow process governed by the magnetic dipole interaction of nuclear magnetic moments, phonons being the main energy sink. As the pressure is raised to a few GPa and the Debye temperature increases substantially, the conversion energy finds itself in an area where phonon states are depleted and conversion slows down. The recent Raman and NMR experiments showed that the conversion rate in $\mathrm{H}_{2}$ after an initial slowdown predicted by theory increases immensely. As for solid $\mathrm{D}_{2}$, conversion rates have apparently not yet been directly measured under pressure. In order to explain the anomaly observed in $\mathrm{H}_{2}$, we have suggested a new conversion mechanism, in which the basic conversion-producing interaction only initiates conversion whereas the energy is removed by rotational excitations via the stronger electric quadrupole-quadrupole interaction. Estimated conversion rates are in good qualitative agreement with available experimental observations. Here we extend the theory to solid $\mathrm{D}_{2}$ taking into account the differences between $\mathrm{H}_{2}$ and $\mathrm{D}_{2}$ in the molecular and solid-state parameters. The new libron-mediated channel is predicted to result for $\mathrm{D}_{2}$ in conversion rates under pressure that are by an order of magnitude larger than at $P=0$.
\end{abstract}

PACS: 61.10.-i, 61.66.-f, 65.70.+y

\section{Introduction}

The requirements of quantum mechanics for a homonuclear hydrogen species $\left(\mathrm{H}_{2}, \mathrm{D}_{2}, \mathrm{~T}_{2}\right)$ rigidly link the rotational momentum $J$ and the total nuclear spin $I$ of the molecule [1]. The states with even $I$ values have even $J$ values and vice versa. The states with the parity of the largest possible $I$ value are called ortho while the states with the other parity are para. Thus, in $\mathrm{H}_{2}$ the states with $I=1$ (and, hence, odd $J$ ) are para; in $\mathrm{D}_{2}$ the states with $I=2$ (and, hence, with $I=0$ too and with even $J$ ) are ortho while those with $I=1$ (and odd $J$ ) are para. Since the energy difference between the $J=1$ and $J=0$ rotational levels is about $170 \mathrm{~K}$ for $\mathrm{H}_{2}$ and $85 \mathrm{~K}$ for $\mathrm{D}_{2}$, only these two states are actually occupied in the solid phase at low temperatures. Transitions between states of different parity ( $J$ or $I$ ), termed ortho-para conversion, are strictly forbidden in a single molecule. The presence of magnetic fields produced by other molecules results in observable conversion rates. At low pressures and low temperatures the conversion process has a low proba- bility, especially in solid deuterium (for conversion rates in $\mathrm{H}_{2}$ and $\mathrm{D}_{2}$ at ambient pressure see Ref. 2). The main conversion-promoting mechanism both in $\mathrm{H}_{2}$ and $\mathrm{D}_{2}$ is the magnetic dipole interaction $\mathcal{H}_{s s}$ between nonzero nuclear spins of two $J=1$ molecules, one of which goes from $J=1$ to $J=0$ state, dissipating the energy into one or two phonons.

With the advent of the modern diamond anvil cell techniques, pressure has become an instrument that is able to drastically change the interplay between different energies in many physical phenomena, one of which is conversion in the hydrogens. The conversion rate in solid $\mathrm{H}_{2}$ slightly increases with pressure, reaches a maximum, then goes down (as predicted by the low-pressure conversion theory [3,4]). But as the pressure is raised above ; $2.5 \mathrm{GPa}$ the conversion rate unexpectedly curves up (see Fig. 1), reaching values by a few orders of magnitude exceeding those observed at moderate pressures (see detailed discussion and the relevant references in Ref. 5). This conversion enhancement in pressurized $\mathrm{H}_{2}$ was first studied in sufficient detail by Raman scattering [6] and then by 


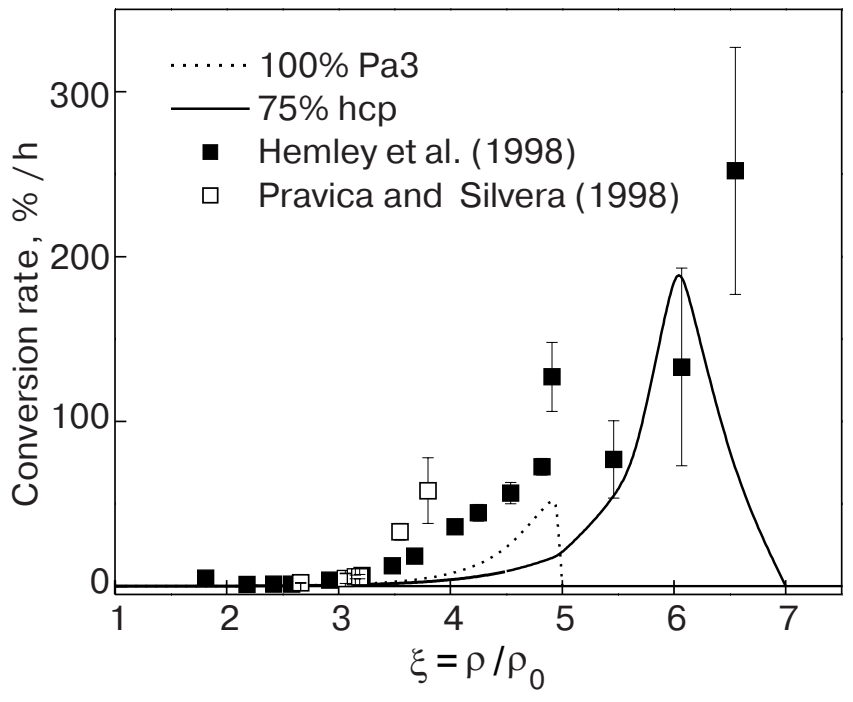

Fig. 1. Measured conversion rates in $\mathrm{H}_{2}$ versus density. The points are from [6] (solid circles) and [7] (empty squares). Rate data for low and moderate pressures are not shown. The curves are semi-quantitative evaluations [10] for $100 \%$ and $75 \%$ ortho fractions.

NMR [7]; indirectly it has been corroborated by Raman scattering in the $\operatorname{Ar}\left(\mathrm{H}_{2}\right)_{2}$ stoichiometric compound [8].

Three factors control the conversion process. One is the interaction that initiates a conversion act. The second one is the agent that carries away the conversion energy $E_{c}$ released during this act. And the third one is the pathway the energy goes from the kinetic rotational form to that determined by the energy sink excitations. The shape of the density of phonon states does not suffer crucial changes [9] under pressure; the width of the phonon energy distribution expands very fast with compression; the energy $E_{c}$ (to be dissipated into phonons) decreases with compression. Due to the combination of these facts, already at relatively moderate pressures, $E_{c}$ finds itself in an energy domain where the density of phonon states is extremely low, rendering the conversion less and less probable. Therefore, given everything else untouched, phonons cannot be the agent for an efficient conversion energy removal at high pressures. It is clear that one has to look for an other type(s) of excitation. The most obvious candidate can be the rotational excitations. Quite simple considerations [10] show that pressure-related changes in the rotational spectrum of mixed ortho-para $\mathrm{H}_{2}$ crystals open up a possibility to dissipate the conversion energy into the rotational energy bath. Below we discuss the new conversion mechanism in some detail.

At low pressures, the conversion mechanisms in solid $\mathrm{D}_{2}$ are known [11]. The ambient-condition conversion rates are known to high accuracy at low temperatures in the solid (cf. Ref. 2) and the calculated conversion constants [11,12] are in good agreement with experiment. Although a certain conversion enhancement in pressurized $\mathrm{D}_{2}$ was reported by Cui et al. [13], no systematic conversion rate measurements have apparently been made in $\mathrm{D}_{2}$ at elevated pressures. The authors know only of one Raman scattering measurement that can give an estimate of the conversion rate $K$ in solid $\mathrm{D}_{2}$ at a pressure of $17 \mathrm{Gpa}$ [14]. The corresponding evaluation, using the known relationship [15] modified for $\mathrm{D}_{2}$, and assuming an exponential variation of the $J=1$ fraction in time (see subsequently sections), gives $K ; 8 \cdot 10^{-3} \mathrm{~h}^{-1}$, which is substantially faster than at zero pressure $(K=$ $=5.6 \cdot 10^{-4} \mathrm{~h}^{-1}$ ) [16]. The main aim of this work is to show that considerations similar to those for dense $\mathrm{H}_{2}$ are applicable for the conversion in $\mathrm{D}_{2}$ at high pressures and that a conversion acceleration should be expected at sufficiently high pressures. The theoretical predictions for conversion rates in solid $\mathrm{D}_{2}$ at high pressures should stimulate further experimental study.

In the next Section we analyze the deuterium molecular parameters and the interactions relevant to the issue of conversion in solid $\mathrm{D}_{2}$ at high densities (comparing them with those in solid $\mathrm{H}_{2}$ ) with an eye for the most efficient mechanisms to be operative at high pressures. We then present (Sec. 3) the current understanding of the reasons behind the pressure-related conversion acceleration in $\mathrm{H}_{2}$. Section 4 deals with a qualitative evaluation of the conversion rates in solid $\mathrm{D}_{2}$ at high pressures for a few of the most promising mechanisms.

\section{Molecular parameters and interactions}

In Table we compile the values of the quantities that are relevant to the conversion in $\mathrm{H}_{2}$ and $\mathrm{D}_{2}$.

Table

The molecular and nuclear parameters of the deuterium and hydrogen molecules; $\mu_{n}=5.05038(36) \cdot 10^{-24} \mathrm{erg} / \mathrm{Gs}$ is the nuclear magneton; $Q$ unit for quadrupole moment times electron charge is $1.3449 \cdot 10^{-26}$ CGSE

\begin{tabular}{c|c|c}
\hline \hline Property (units) & $\mathrm{D}_{2}$ & $\mathrm{H}_{2}$ \\
\hline \hline$\mu\left(\right.$ in $\left.\mu_{n}\right)$ & $0.8574073[17]$ & $2.79245(2)[18]$ \\
$\mu_{\text {rot }} / J\left(\right.$ in $\left.\mu_{n}\right)$ & $0.44288(52)[19]$ & $0.88291(7) *[20]$ \\
$Q_{N}\left(10^{-27} \mathrm{~cm}^{-2}\right)$ & $2.738(16)[22]$ & - \\
$Q(Q$ units $)$ & $0.48529[23]$ & $0.47702[23]$ \\
$E(J=1)\left(\mathrm{cm}^{-1}\right)$ & $59.7804[24]$ & $118.495[25]$ \\
$E(J=2)\left(\mathrm{cm}^{-1}\right)$ & $179.065[24]$ & $354.39[25]$ \\
\hline \hline Consistency of the relationship $\mu_{\operatorname{rot}}(J=2) ; 2 \mu_{\operatorname{rot}}(J=1)$ was
\end{tabular}


There are three basic interactions that can change the parity of the total nuclear spin of a $\mathrm{D}_{2}$ or $\mathrm{H}_{2}$ molecule that is supposed to convert [11]:

(A) Interaction of the magnetic nuclear moment of the chosen molecule with the nonzero nuclear spin of a neighbor molecule.

(B) Interaction of the same chosen nuclear magnetic moment with the effective magnetic moment of the $J=1$ state of a proper neighbor.

(C) Interaction of the nuclear electrical quadrupole moment of the chosen molecule (nonzero for $I \geq 1$ ) with the electric field gradient due to the quadrupole moment of the $J=1$ rotational state of a proper neighbor.

Interaction (A) is operative not only when the conversion-promoting neighbor has $I=1$, i.e., is in the $J=1$ state (case A1) but also when $I=2$, i.e., when the promoter is in a state with $J=0$ (case A2). The former interaction (A1) is a direct analog of the corresponding interaction in $\mathrm{H}_{2}$, whereas the latter (A2) is inherent only in $\mathrm{D}_{2}$. Both terms can be written in the same form

$$
\mathcal{H}_{s s}=-4 \sqrt{6} \mu^{2} \sum_{\sigma, \sigma^{\prime}= \pm 1}\left(\left\{\mathbf{S}_{\sigma} \otimes \mathbf{S}_{\sigma^{\prime}}\right\}_{2} \cdot \mathbf{C}_{2}\left(\mathbf{n}_{\sigma \sigma^{\prime}}\right)\right) R_{\sigma \sigma^{\prime}}^{-3}
$$

Here $\mu$ is the nuclear magnetic moment of the deuteron (proton); $\mathbf{R}_{\sigma \sigma^{\prime}}=\mathbf{R}_{0}+(d / 2)\left(\mathbf{w}^{\prime} \sigma^{\prime}-\mathbf{w} \sigma\right)=R_{\sigma \sigma^{\prime}} \mathbf{n}_{\sigma \sigma^{\prime}} ;$ $\mathbf{R}_{0}$ is the radius vector between the two molecular centers; $\sigma$ and $\sigma^{\prime}$ refer to the respective deuterons (protons) respectively in the primed and un-primed molecules; $\mathbf{S}_{\sigma}$ is the nuclear spin operator of deuteron (proton) $\sigma ; d$ is the interatomic distance in the molecule; $\mathbf{w}$ and $\mathbf{w}^{\prime}$ are the unit vectors along the respective molecular axes; $\{\mathbf{A} \otimes \mathbf{B}\}_{N}$ and $\left(\mathbf{A}_{M} \cdot \mathbf{B}_{M}\right)$ denote respectively direct and scalar products [26] of two irreducible tensors (in the latter case, of the same rank).

Interaction (B) is another direct analog of the respective interaction in solid $\mathrm{H}_{2}$ with the difference that the effective value of $\mu_{\text {rot }}$ in $D_{2}$ is appreciably larger than in $\mathrm{H}_{2}$ (see Table).

Interaction (C) can be represented by the following Hamiltonian

$$
\begin{gathered}
\mathcal{H}_{q}=3 \sqrt{70} e^{2} Q Q_{N} \times \\
\times \sum_{i}\left(\left\{\mathbf{C}_{2}\left(\mathbf{S}_{i}\right) \otimes \mathbf{C}_{2}\left(\mathbf{w}^{\prime}\right)\right\}_{4} \cdot \mathbf{C}_{4}\left(\mathbf{n}_{i}\right)\right) R_{i}^{-5} .
\end{gathered}
$$

Here $Q$ is the quadrupole moment of the deuterium molecule in the $J=1$ state; $Q_{N}$ is the nuclear quadrupole moment of the deuteron; $\mathbf{R}_{i}=R_{i} \mathbf{n}_{i}$ is the distance between deuton $i$ in the chosen molecule and the center of the conversion-promoting para neighbor; other notation as in Eq. (1).
Since the orientational interactions play an important role at high pressures, the above two Hamiltonians should be complemented by the electric quadrupole-quadrupole (EQQ) interaction energy

$$
\begin{gathered}
\mathcal{H}_{E Q Q}=\sqrt{\frac{35}{2}}(e Q)^{2} \times \\
\times \sum_{i j}\left(\left\{\mathbf{C}_{2}\left(\mathbf{w}_{i}\right) \otimes \mathbf{C}_{2}\left(\mathbf{w}_{j}\right)\right\}_{4} \cdot \mathbf{C}_{4}\left(\mathbf{n}_{i j}\right)\right) R_{i j}^{-5}
\end{gathered}
$$

where $i$ and $j$ numerate lattice sites occupied by $J=1$ molecules.

The macroscopic equations for the $J=1$ fraction $x$ are different for the two hydrogen isotopes under consideration. In $\mathrm{H}_{2}$, both operating conversion-promoting interactions result in a second-order reaction equation

$$
\frac{d x}{d t}=K x^{2} \text { for hydrogen }
$$

where the conversion parameter $K$ can depend on compression and, generally, on $x$. At low pressures, $K$ is virtually a constant $K_{0}$ and Eq. (4) has the solution ( $x_{0}$ being the ortho fraction at $\left.t=0\right): 1 / x=K_{0} t$. In $\mathrm{D}_{2}$, both $J=1$ and $J=0$ contribute to the conversion probability as promoters, so that the respective equation for the $J=1$ fraction is

$$
\frac{d x}{d t}=-k x^{2}-k^{\prime} x(1-x) \text { for deuterium }
$$

At zero pressure the values of $k$ and $k^{\prime}$ are very close so that, effectively, Eq. (5) yields an exponential decay dependence $x(t) \propto \exp (-k t)$.

\section{Conversion in solid $\mathrm{H}_{2}$ at high pressure}

We briefly summarize the main causes that lead to the dramatic conversion acceleration in solid $\mathrm{H}_{2}$ at high pressures. As shown above, phonons cease to be efficient as conversion energy sink at comparatively moderate pressures. The main idea for a consistent explanation of the pressure-related conversion enhancement was to consider orientational (rotational) degrees of freedom. There are a few problems to be solved for a successful implementation of that idea: (i) the particular conversion channel (energy path, type of conversion act, sink excitations); (ii) the shape of the rotational energy spectrum in such a highly random system as an orientationally disordered orthopara mixture; and (iii) the compression-induced variation of the said spectrum.

The path mainly responsible for this acceleration [10] is as follows (Fig. 2). The Hamiltonian $\mathcal{H}_{s s}$ starts conversion, producing a non-equilibrium intermediate state, from which the system goes to equilibrium through the stronger EQQ interaction and emits 


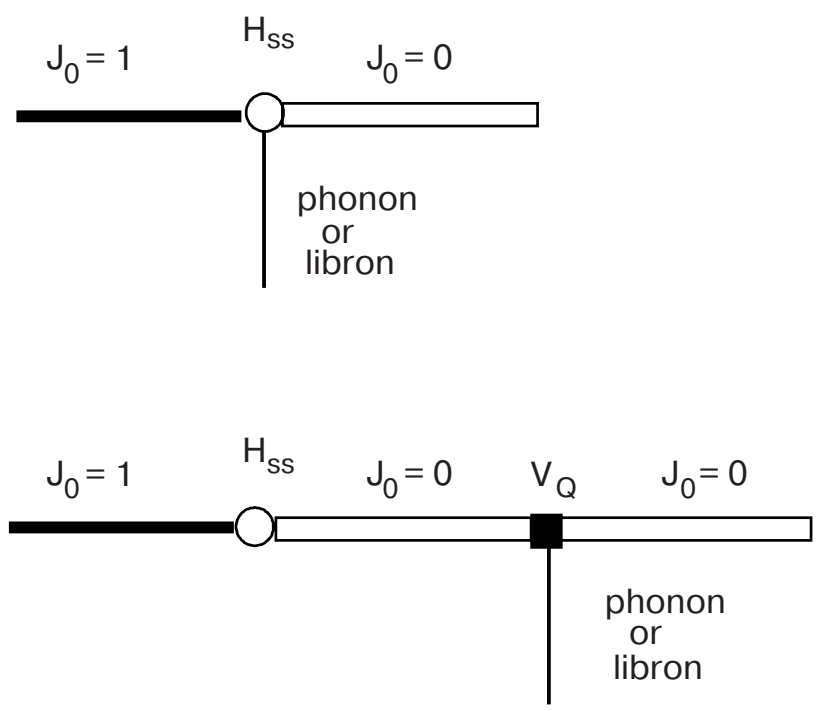

Fig. 2. Vertices for direct (standard) pathway and a pathway with intermediate state. For standard channels (upper diagram), $\mathcal{H}_{s s}$ starts conversion and produces sink excitations. In channels with intermediate states, $\mathcal{H}_{s s}$ only starts conversion whereas excitations are created through the stronger EQQ interaction.

a rotational excitation. This path becomes efficient only at a sufficiently high pressure [5].

Proceeding from the available experimental evidence we argue $[5,27]$ that, given a large enough $J=1$ concentration, the rotational spectrum of a mixed crystal basically resembles that of an orientationally ordered state: a low-energy maximum containing local ground states and a maximum at higher energies that corresponds to local but collectivized excited rotational states.

Evolution of the rotational spectrum with compression is schematically represented in Fig. 3. Because of the normalization employed the plot is valid both for $\mathrm{H}_{2}$ or $\mathrm{D}_{2}$. Though being directly applicable to a $100 \%$ $J=1$ orientationally ordered solid, the reasoning of the preceding paragraph makes the plot qualitatively valid for sufficiently high $J=1$ concentrations even in the disordered state. A very important feature is the pressure-related decrease in the conversion energy due to the growing strength of the negative molecular-field offset.

Calculation of the conversion rate at high pressure is impossible in analytical form; using semi-quantitative evaluations [10], we obtained results (the curves in Fig. 1) that are consistent with the experimental findings. It was later shown [5] that other channels, though bringing about considerable changes (mostly at moderate pressures), do not contribute appreciably to the pressure-related conversion enhancement. The new theory predicts a few effects, some of which find

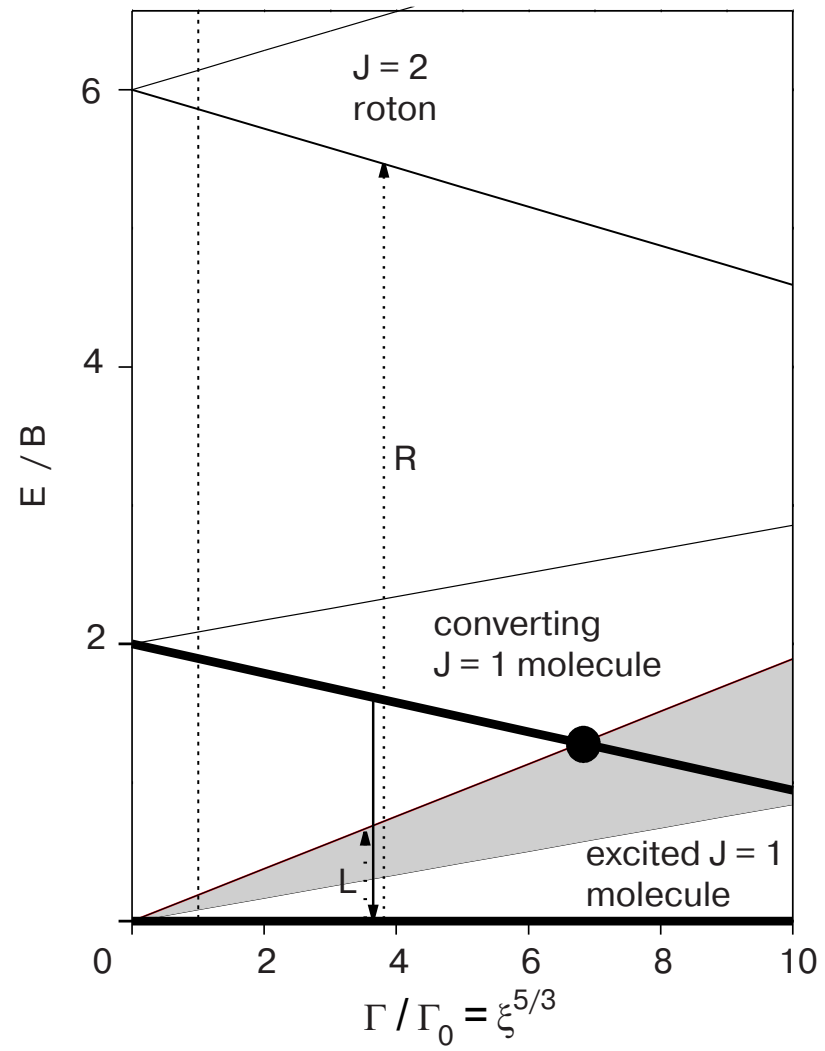

Fig. 3. Energy scheme (applicable both for $\mathrm{H}_{2}$ and $\mathrm{D}_{2}$ ) for conversion transitions involving $J=1$ and $J=2$ excitations with varying EQQ interaction. The $J=2$ roton (arrow R) is too energetic to take up the conversion energy. The energy floor is the state in which the chosen molecule has converted and all other $J=1$ molecules are in their local rotational ground states. During conversion the chosen molecule starts from a state with the energy to be released (solid down-pointing arrow). Another ortho molecule can now be excited to the libron band (shaded region) to take up the energy (shown as a broken up-pointing arrow $L$ ). At a critical $\Gamma$ value (large circle) the energy span of the excited molecule is wide enough to accommodate the entire conversion energy.

confirmation in the available experimental results. Unlike at low pressures, the conversion rate $K$ should be a strong function of the running $J=1$ fraction $x$. Analysis of the $x$ versus time curves [5,6] for sufficiently high pressures shows (Fig. 4) that, indeed, the conversion rate considerably decreases in time (or with decreasing $J=1$ fraction).

\section{Conversion in solid $\mathrm{D}_{2}$ at high pressure}

Turning now to the efficiency of various conversion channels in solid deuterium at high pressures we can make use of some of the formulas derived for the case of $\mathrm{H}_{2}$ [5] by allowing for the differences in the molecular parameters. All of the standard channels (i.e., 


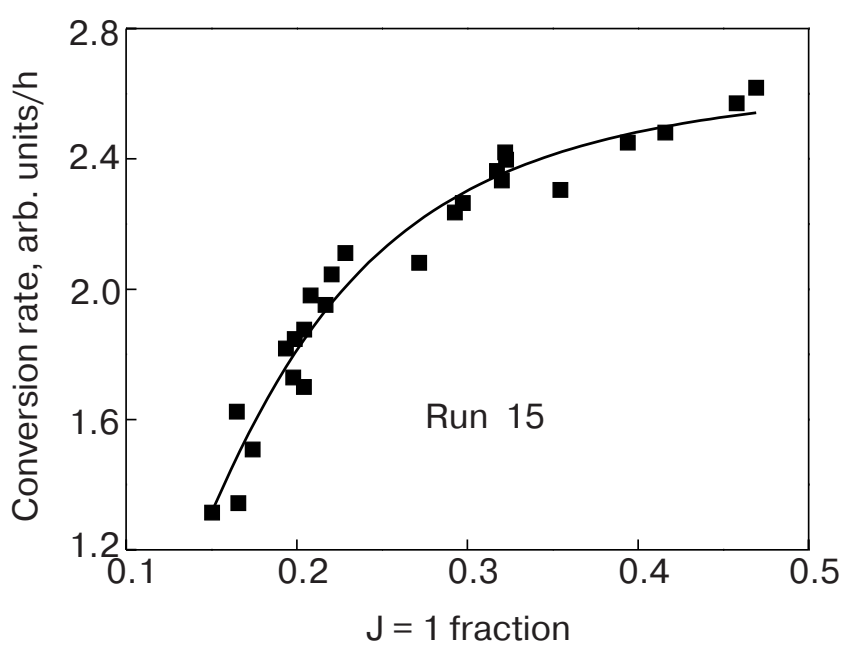

Fig. 4. The conversion rate $\mathrm{K}$ in $\mathrm{H}_{2}$ as a function of the running $J=1$ fraction for a pressure of $47.7 \mathrm{GPa}$, as reconstructed from Raman scattering measurements [6]. The drop in $K$ is more than two-fold.

those without an intermediate state) are phonon-assisted and are therefore inefficient at high pressures. The idea of the intermediate state with subsequent participation of the EQQ interaction is productive for $\mathrm{D}_{2}$ as well. Without going into detailed calculations for channels with intermediate state, which will be published elsewhere, we give a quantitative description of what can be expected for conversion rates in compressed $\mathrm{D}_{2}$. Interaction A1 (cf. Sec. 2) will yield the same result as for $\mathrm{H}_{2}$ but only for the conversion parameter $k$ in Eq. (5), viz., (cf. [10])

$$
k_{A 1} \propto \frac{\xi^{13 / 3}}{\left[E_{c}(\xi)\right]^{2}} g_{0}(\varepsilon) .
$$

Here $\xi=\rho / \rho_{0}$ is the crystal density ratio reduced to the $P=0$ value; $E_{c}$ is the conversion energy as a function of $\xi$ (see Fig. 3 ) and $g_{0}(\varepsilon)$ is the density of rotational (libron-like) states. The contribution due to interaction A2, which goes to the conversion parameter $k^{\prime}$, in analytical form is similar to Eq. (6). Interaction $\mathrm{B}$ gives a contribution to $k$ of the same form as in Eq. (6). Finally, interaction $\mathrm{C}$ contributes also to $k$ but, originating from the energy with a different dependence on the separation $R$ [compare Eqs. (1) and (2)], is different as a function of compression $\xi$ :

$$
k_{C} \propto \frac{\xi^{19 / 3}}{\left[E_{c}(\xi)\right]^{2}} g_{0}(\varepsilon) .
$$

As mentioned above, at zero pressure the constants $k$ and $k^{\prime}$ are virtually the same, that is, $k_{A 1}+k_{B}+$ $+k_{C} ; k_{A 2}^{\prime}$. As the pressure is increased to the level in which the channels with intermediate state become efficient, this match will be lifted and the time de- pendence of the $J=1$ concentration will cease to be exponential.

As it was explained above, Fig. 3 is plotted in reduced variables such that it is valid both for $\mathrm{H}_{2}$ and $\mathrm{D}_{2}$. However, when replotting in absolute values ( $J=1$ energies, pressures, compressions $)$ it will look different for the two isotopes. It should be also noted that Fig. 3 is rigorously applicable only for pure $J=1$ solids; lower $x$ necessitate recalculation of, for example, the position of the critical point (solid circle in Fig. 3) where rotational excitations come into play. The quantities we need for re-scaling to absolute values are the volume dependence of the Debye frequency [28] and the equation of states (EOS). Fortunately, at high pressures the equation of state for $\mathrm{H}_{2}$ and $\mathrm{D}_{2}$ are almost the same [29], the differences are appreciable only at rather low pressures. The corresponding estimations give the following critical pressure values. For pure para $(J=1)$ deuterium the indirect channel will become efficient at a pressure of $1.5 \mathrm{GPa}$ and cease to operate around $4.8 \mathrm{GPa}$; for normal $(33 \%$ para) deuterium these two pressure values are $14 \mathrm{GPa}$ and $32 \mathrm{GPa}$. We note that the working range for normal $\mathrm{D}_{2}$ extends beyond the critical pressure (28 GPa) [30], at which $\mathrm{D}_{2}$ transforms to the broken-symmetry phase (BSP). This means that at these pressures the amount of the admixed $J=2$ state should be appreciable. As can be seen from Table, the effective magnetic moment of this state is large enough to change noticeably the strength of the total conversion-initiating interaction. This problem requires a special consideration, preferably with the structure of the BSP known.

Since the zero-pressure conversion energy is less than the deuterium Debye temperature, the principal

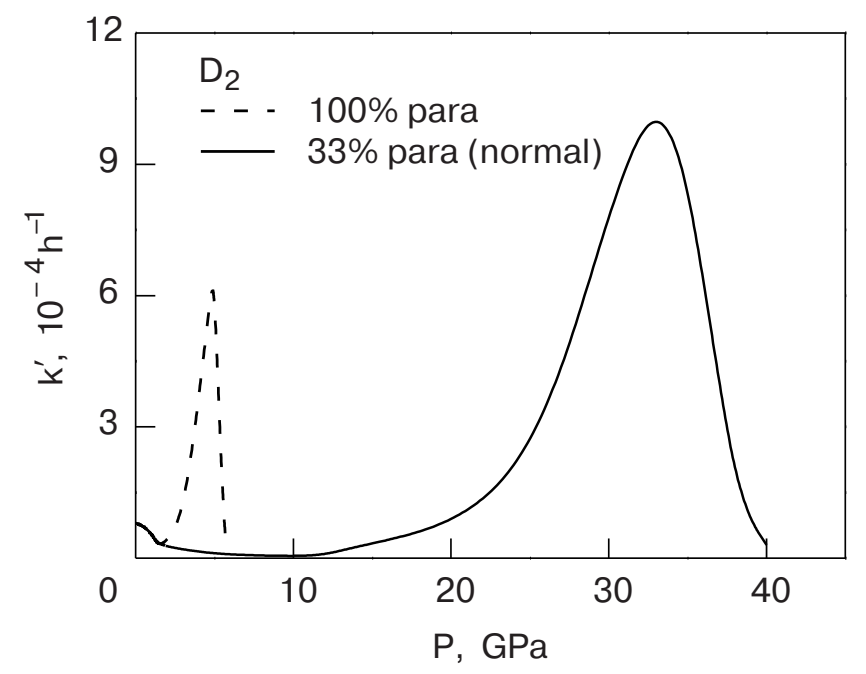

Fig. 5. The conversion parameter $k^{\prime}$ in $\mathrm{D}_{2}$ as a function of pressure, as predicted in this report. 
conversion mechanism at $P=0$ is a one-phonon one (which is not so in $\mathrm{H}_{2}$ ). Therefore, the phonon-mediated conversion rate as a function of pressure will have no distinct maximum at low pressures but will start to decrease on initial compression and at a comparatively low pressures (if the para fraction is high) will start building up but to lower final values compared to $\mathrm{H}_{2}$. A schematic representation of this dependence is shown in Fig. 5.

\section{Acknowledgments}

This work was supported by the CRDF (grant UP2-2445-KH-02) and NSF/DMR. The authors thank A. F. Goncharov for providing his unpublished results. M.A.S. also thanks Irina Legchenkova for technical assistance.

1. J. Van Kranendonk, Solid Hydrogen, Plenum, New York (1983)

2. V.G. Manzhelii and M.A. Strzhemechny, Quantum Molecular Crystals, in: Physics of Cryocrystals, V.G. Manzhelii, Yu.A. Freiman, M.L. Klein, and A.A. Maradudin (eds.), AIP Publishers, Woodbury (1997).

3. K. Motizuki and T. Nagamiya, J. Phys. Soc. Jpn. 11, 93 (1956).

4. A.J. Berlinsky and W. N. Hardy, Phys. Rev. B8, 5013 (1973).

5. M.A. Strzhemechny, R.J. Hemley, H.K. Mao, A.F. Goncharov, and J.H. Eggert, Phys. Rev. B66, 014103 (2002).

6. R.J. Hemley, A.F. Goncharov, H.K. Mao, E. Karmon, and J.H. Eggert, J. Low Temp. Phys. 110, 75 (1998).

7. M.G. Pravica and I.F. Silvera, Phys. Rev. Lett. 81, 4180 (1998)

8. F. Grazzi and L. Ulivi, Europhys. Lett. 52, 564 (2000).

9. S.B. Feodosyev, I.A. Gospodarev, M.A. Strzhemechny, and R.J. Hemley, Physica B300, 186 (2001).

10. M.A. Strzhemechny and R.J. Hemley, Phys. Rev. Lett. 85, 5595 (2000).

11. K. Motizuki, J. Phys. Soc. Jpn. 12, 163 (1957).
12. M.A. Strzhemechny and O.I. Tokar, Fiz. Nizk. Temp. 11, 813 (1985) [Sov. J. Low Temp. Phys. 11, 446 (1985)].

13. L. Cui, N.H. Chen, and I.F. Silvera, Phys. Rev. B51, 14987 (1995).

14. A.F. Goncharov, private communication.

15. I.F. Silvera, Rev. Mod. Phys. 52, 393 (1980).

16. Yu.Ya. Milenko and R.M. Sibileva, Fiz. Nizk. Temp. 1, 796 (1975) [Sov. J. Low Temp. Phys. 1, 382 (1975)].

17. N.F. Ramsey, Molecular Beams, Clarendon Press, Oxford (1956)

18. F. Bloch and C.D. Jeffries, Phys. Rev. 80, 305 (1950)

19. G.G. Barnes, P.J. Bray, and N.F. Ramsey, Phys. Rev. 94, 893 (1954)

20. N.J. Harrick and N.F. Ramsey, Phys. Rev. 88, 228 (1952)

21. N.F. Ramsey, Phys. Rev. 58, 226 (1940).

22. H.G. Kolsky, T.E. Phipps, N.F. Ramsey, and H.B. Silsbee, Phys. Rev. 87, 395 (1952).

23. G. Karl and J.D. Poll, J. Chem. Phys. 46, 2944 (1967)

24. A.R.W. McKellar and T. Oka, Can. J. Phys. 56, 1315 (1978)

25. A.P. Stoicheff, Can. J. Phys. 35, 730 (1957).

26. D.A. Varshalovitch, A.N. Moskalev, and V.K. Khersonskii, Theory of Angular Momentum, World Scientific, Singapore (1988).

27. M.A. Strzhemechny, Proc. NATO ARW, Frontiers of High Pressure Research II: Application of High Pressure to Low-Dimensional Novel Electronic Materials, Pingree Park, CO, USA, 10-15 June 2001, H.D. Hochheimer, B. Kuchta, P.K. Dorhout, and J.L. Yarger (eds.), NATO Series II, Kluwer Academic 48, 235 (2001).

28. R.J. Hemley, J.H. Eggert, and H.K. Mao, Phys. Rev. B48, 5779 (1993).

29. P. Loubeyre, R. LeToullec, D. Hausermann, M. Hanfland, R.J. Hemley, H.K. Mao, and L.W. Finger, Nature 383, 702 (1996).

30. I.F. Silvera and R.J. Wijngaarden, Phys. Rev. Lett. 47, 39 (1981). 